\title{
Fauna of Odonata of the Adygheya Republic (North-Western Caucasus)
}

\author{
Фауна стрекоз (Odonata) Респубцики Адыгея \\ (Северо-Западный Кавказ)
}

\author{
Maksim I. Shapovalov, Erik A. Korotkov \\ М.И. Шаповалов, Э.А. Коротков
}

\begin{abstract}
Laboratory for Bioecological Monitoring of the Invertebrate Animals of Adygheya, Research Institute of Complex Problems, Adyghe State University, Gagarina str. 13, Maykop 385000, Adygheya Republic, Russia. E-mails: shapmaksim2017@yandex.ru, korotkov-erik@mail.ru Лаборатория биоэкологического мониторинга беспозвоночных животных Адыгеи, НИИ Комплексных проблем, Адыгейский государственный университет, Гагарина 13, Майкоп 385000, Республика Адыгея, Россия.
\end{abstract}

KEY WORDS: fauna, dragonfly, annotated checklist, Republic of Adygheya, North-Western Caucasus, Odonata.

КЛЮЧЕВЫЕ СЛОВА: фауна, стрекозы, аннотированный список, Республика Адыгея, Северо-Западный Кавказ, Odonata.

ABSTRACT. An annotated checklist of Odonata of the Adygheya Republic (Russia) is given. It includes 43 species of eight families: Calopterygidae (2 species), Coenagrionidae (10), Platycnemidae (1), Lestidae (6), Aeshnidae (8), Corduliidae (1), Gomphidae (3) and Libellulidae (12). New collecting sites are given for 39 species. Coenagrion scitulum (Rambur, 1842), Erythromma najas najas (Hansemann, 1823), Pyrrhosoma nymphula (Sulzer, 1776), Onychogomphus flexuosus (Schneider, 1845), Cordulia aenea (Linnaeus, 1758), Orthetrum cancellatum (Linnaeus, 1758), O. coerulescens anceps (Fabricius, 1798), Sympetrum fonscolombii (Selys, 1840) and Crocothemis erythraea (Brulle, 1832) are described for the first time for the Republic of Adygheya.

РЕЗЮМЕ. В статье даётся обобщённый список стрекоз (Odonata) Республики Адыгея, включающий 43 вида из 8 семейств: Calopterygidae (2 вида), Coenagrionidae (10), Platycnemidae (1), Lestidae (6), Aeshnidae (8), Corduliidae (1), Gomphidae (3) и Libellulidae (12). Для 39 видов приводятся новые пункты сборов. Coenagrion scitulum (Rambur, 1842), Erythromma najas najas (Hansemann, 1823), Pyrrhosoma nymphula (Sulzer, 1776), Onychogomphus flexuosus (Schneider, 1845), Cordulia aenea (Linnaeus, 1758), Orthetrum cancellatum (Linnaeus, 1758), $O$. coerulescens anceps (Fabricius, 1798), Sympetrum fonscolombii (Selys, 1840), Crocothemis erythraea (Brulle, 1832) впервые указываются для территории Адыгеи.

\section{Introduction}

The odonatology of the North-Western Caucasus has been studied from the beginning of the 20th century. A. Brauner [1903] was the first researcher who published a study on the Odonata of Caucasus. He published an annotated checklist of species collected between Novorossiysk and Adler. For the first time, A.N. Bartenev [1910] recorded 34 dragonflies species for the NorthWestern Caucasus, based on the material collected in the southwestern Kuban Oblast: Libellulidae (11 species), Coenagrionidae (6), Aeschnidae (6), Lestidae (5), Corduliidae (2) and Calopterygidae (2). Later, Bartenev [1929] described several dragonflies taxa of the NorthWestern Caucasus, based on the material from the montane lakes "Seen auf dem Berge Atshischgho", or "Seen am Berge Atshischgho in der Umgebung von Krasnaja Poljana" _ "lakes on the Achishkho mountain". The described odonates were Leucorrhinia circassica Bartenev, 1929, Enallagma rotundatum Bartenev, 1929, and Aeshna juncea atshischgho Bartenev, 1929.

The fauna of Odonata of the Western Caucasus, including the Caucasus Nature Reserve, is summarized in the study "Materials to the knowledge of Odonata of the Western Caucasus" that describes 58 species [Bartenev, 1930a]. In a review on the Palearctic species, the genus Cordulegaster was described from the upper reaches of the Mzymta River (environs of Sochi) as the species Cordulegaster mzymtae. The description was based on one female specimen without 7-10 abdominal segments and apical parts of the wings [Bartenev, 1930b]. 
Y.M. Kolosov [1915] recorded the species Sympetrum depressiusculum Selys, Anax parthenope Selys, and Erythromma najas Hans. in the environs of Yekaterinodar in his small note on the addition to the odonatan fauna of the Kuban Oblast.

V.Y. Stain [2002, 2003a, b, etc.] studied zoogeography, ecology, and biology of dragonflies of the NorthWestern Caucasus region. The fauna of dragonflies of Caucasian Riviera inside Krasnodar Krai was studied by O.E. Kosterin [Kosterin, Solovyev, 2017; Kosterin, 2017a, b, 2018].

According to zoogeographical regions of the North Caucasus (based on the Odonata material), the Adygheya Republic belongs to the Maykop Okrug, western Caucasian subprovince, the fauna of whose includes 36 species [Stain, 2002, 2003b]. The dragonflies fauna of the Adygheya Republic has not been studied comprehensively, there are only a few insufficient studies of this region [Bartenev, 1910, 1930a; Mayzel, 1940; Shapovalov, Korotkov, 2018]. A summary on the dragonflies of the Adygheya Republic has never been published, which shows the importance of our study.

The Adygheya Republic is a region with zones of intensive agriculture, urbanized landscapes, and almost unchanged montane territories. The Adygheya Republic is located in the central area of the North-Western Caucasus, a basin of the Kuban, Laba, and Belaya Rivers, between $45^{\circ} 13^{\prime} \mathrm{N}$ and $43^{\circ} 46^{\prime} \mathrm{N}, 38^{\circ} 41^{\prime} \mathrm{E}$ and $40^{\circ} 30^{\prime} \mathrm{E}$. The northern part of the republic is located on the inclined terraced Zakubanskaya plain, with the minimum altitude in the environs of the Pslukh aul $(20 \mathrm{~m}$ a.s.l.) and the maximum altitude on the south-east $(300$ $\mathrm{m}$ a.s.1). The Zakubanskaya plain is divided by river valleys, tributaries of the middle and lower Kuban. The ranges of the Greater Caucasus and its spurs are located on the 1/3 of the Adygheya Republic. To the south of Maykop there are Lesisty (the highest point is in the Adygheya Republic, 742 m a.s.1.), Pastbishny (the highest point is the Fiziabgo mountain, $992 \mathrm{~m}$ a.s.1.), and Skalisty ranges [Atlas ..., 2001]. Annual precipitation in the Adyghe Republic increases from the north to the south with the increase of altitudes of the Caucasian mountains that play a role of a barrier where precipitation falls down. The annual precipitation is $550-700$ $\mathrm{mm}$ on the plains of the Adygheya Republic, 700-800 $\mathrm{mm}$ in the foothills with altitudes up to $100 \mathrm{~m}$, and 1000-2000 $\mathrm{m}$ and more in the mountains [Varshanina, Mitusov, 2005].

In this study, taking previous literature into account, an annotated checklist of Odonata of the Adygheya Republic, which includes 43 species of 21 genera of 8 families, is given.

\section{Material and methods}

Adult dragonflies were collected in the Adygheya Republic and adjacent regions of Krasnodar Krai in 2007 and 2011-2018 years (Fig. 1). The material was collected by an entomological net [Golub et al., 2012].
Most of the material was mounted using entomological pins, and the other part placed on cotton wool in the Laboratory of Bioecological Monitoring of Invertebrate Animals of Adygheya, Research Institute of Complex Problems, Adyghe State University (Maykop, Russia). The collecting sites were mapped using Qgis Lion 2.04 software, with squares of $5 \mathrm{~km}^{2}$ bound to a coordinate system. Photographs of species were taken using a Canon PowerShot A580 camera, photographs of morphological traits of odonates were taken using Micromed MC-3 ZOOM Led and Micromed-3 var. 3-20 stereo microscopes.

The dragonflies were identified using mostly the following studies: Ketenchiev, Kharitonov [1998], Skvortsov [2010], Kalkman [2006] etc.

The annotated checklist includes previously described in the literature species and new studied material.

\section{Results}

Annotated checklist of Odonata of the Adyghe Republic

\section{ZYGOPTERA Family Calopterygidae}

1. Calopteryx splendens (Harris, 1780)

Shapovalov, Korotkov, 2018: Krasny most settlement, Botanical Garden at Adyghe State University.

MATERIAL. Maykop: Zelenstroy City District, 2.VII.2018 $10^{7}$. Maykopsky District: environs of the Kalinin, 27.VI.2017 $3 \sigma^{7}, 3$; Khanskaya, 6.VI.2012 - $10^{7} ; 2 \mathrm{~km}$ to the Tulsky, canyon of the Maykopskaya River, 2.VIII.2017 - 10'; environs of the Krasny most, Botanical Garden at Adyghe State University, 13.VI.2017 $10^{7}$; 8.VI.2018 - 20'; 14.VI.2018 - 19. Giaginsky District: Trud settlement, Ayryum River, 17-19.VII.2011 - 70', 5+; Giaginskaya,

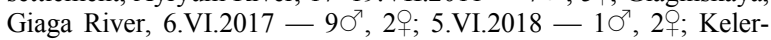
messkaya, 5.VI.2018 - 10'. Koshekhablsky District: environs of the Khodz, 5.VII.2017 - 50', 1우 9.VI.2018 - 1; environs of the Volnoye, 4.VI.2015 - 19; 9.VI.2016 - 10'. Shovgenovsky District: environs of the Vesyoly, 14.V.2018 - $60^{2}, 4$.

At the adjacent territory of Krasnodar Krai, Mostovsky District, environs of the Severny, Chekhrak River, 19.VI.2012 - $60^{7}$

REMARKS. C. splendens is represented by a nominotypical subspecies of the Adygheya Republic.

\section{Calopteryx virgo (Linnaeus, 1758)}

Bartenev, 1930a: Maykop, across the Belaya River (as Calopteryx virgo festiva var. feminalis Bart.).

MATERIAL. Maykopsky District: $2 \mathrm{~km}$ to the Tulsky, canyon of the Maykopskaya River, 2.VIII.2017 - 70', 6 ㅇ.

REMARKS. Calopteryx virgo L. is represented by the subspecies $C$. virgo festiva (Brullé, 1832) in most of the Caucasus [Ketenchiev et al., 2016]. A subspecies Calopteryx virgo feminalis Kosterin is distinguished in populations of $C$. virgo of the Caucasian Riviera [Kosterin, 2017b]. Collected individuals from the Adyghe Republic have traits of the subspecies Calopteryx virgo feminalis.

\section{Family Coenagrionidae}

3. Coenagrion ornatum (Selys, 1850) Sel.).

Bartenev, 1910, 1930a: Kelermesskaya (as Agrion ornatum

MATERIAL. Maykopsky District: Khanskaya, 16.VI.2013 $50^{\top}, 3$ ㅇ. 
4. Coenagrion puella (Linnaeus, 1758)

Bartenev, 1930a: Maykop, Kurdzhips River (as Agrion puella L.); Shapovalov, Korotkov, 2018: Krasny most, Botanical Garden at Adyghe State University.

MATERIAL. Maykop: Zelenstroy City District, 10.VI.2016 10'; 31.V.2017 - 20', 1; 11.VI.2017 - 10'; Voskhod City District, 1.V.2018 - 10 ; 7.VI.2018 - 1오. Maykopsky District: environs of the Krasny most, Botanical Garden at Adyghe State University, 13.VI.2017 - 10 ; 24.VI.2017 - 10 ; 8.VI.2018 $10^{7}$. Giaginsky District: Kelermesskaya, Giaga River, 5.VI.2018 - 10' Giaginskaya, Giaga River, 1.V.2018 - $10^{\prime}$, 1; 5.VI.2018 - 1ㅇ․ Shovgenovsky District: environs of the Vesyoly, Ulka River, 14.V.2018 - $20^{7}$. Koshekhablsky District: Volnoye, 9.VI.2016 - $10^{7}$.

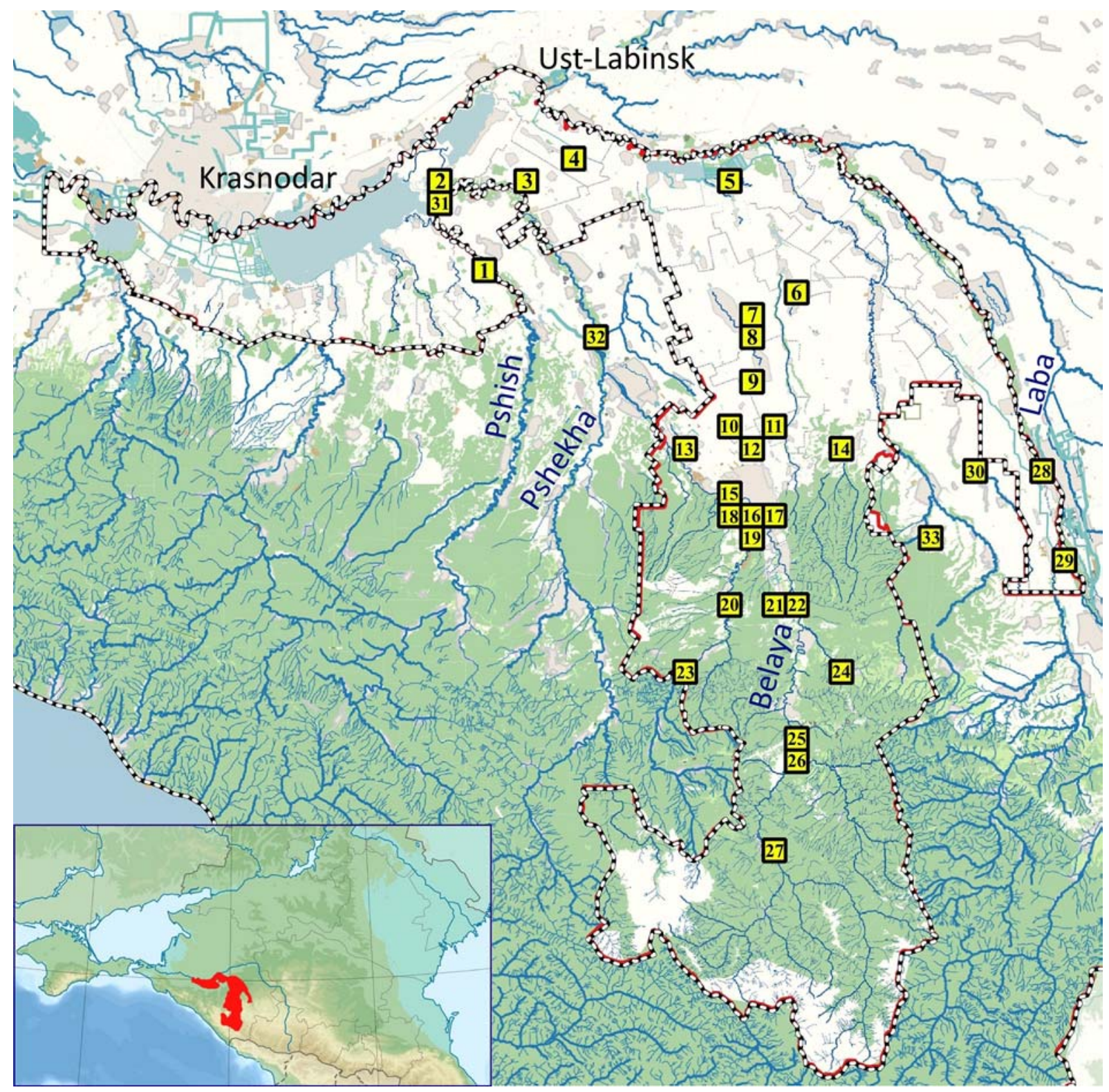

Fig. 1. Collecting sites of the Adygheya Republic and adjacent territories of Krasnodar Krai: 1 - Gabukay, 2 - Adamy, 3 - Beloye, 4 - Elenovskoye, 5 - Vesyoly, Ulka River, 6 - Trud, Ayryum River, 7, 8 - Giaginskaya, 9 - Kelermesskaya, 10 - Podgorny, 11 Kalinin, 12 - Kosinov, 13 - Khanskaya, 14 - Kuzhorskaya, 15 - Maykop, Zelenstroy City District, 16 - Maykop, «Mezdakh», 17 Maykop, Voskhod City District, 18 - Krasnooktyabrsky, 19 - Krasny most, Botanical Garden at Adyghe State University, 20 Kurdzhipskaya, 21 - Shuntuk, 22 - canyon of the Maykopskaya River, 23 - Krasny Dagestan, 24 - Sevastopolskaya, 25, 26 Dakhovskaya, 27 - Khamyshki, 28 - Volnoye, 29 - Khodz, Khodz River, 30 - Severny, Chekhrak River, 31 - Belyaevsky, 32 Verkhnevedeneevsky, 33 - Makhoshevskaya.

Рис. 1. Пункты сборов материала на территории Республики Адыгея и сопредельных территориях Краснодарского края: 1 Габукай, 2 - Адамий, 3 - Белое, 4 - Еленовское, 5 - Весёлый, р. Улька, 6 - Труд, р. Айрюм, 7, 8 - Гиагинская, 9 - Келермесская, 10 - Подгорный, 11 - Калинин, 12 - Косинов, 13 - Ханская, 14 - Кужорская, 15 - Майкоп, p-н Зеленстрой, 16 - Майкоп, pн Мэздах, 17 - Майкоп, р-н Восход, 18 - Краснооктябрьский, 19 - Красный мост, Ботсад АГУ, 20 - Курджипская, 21 - Шунтук, 22 - ущ. р. Майкопская, 23 - Красный Дагестан, 24 - Севастопольская, 25, 26 - Даховская, 27 - Хамышки, 28 - Вольное, 29 - Ходзь, р. Ходзь, 30 - Северный, р. Чехрак, 31 - Беляевский, 32 - Верхневеденеевский, 33 - Махошевская. 
At the adjacent territory of Krasnodar Krai, Belorechensky District: environs of the Verkhnevedeneevsky, 14.VI.2012 - $10^{\prime}$, 1․ Apsheronsky District: 3 km N Krasny Dagestan, Kurdzhips River, 21.VI.2012 - $10^{7}$.

REMARKS. Previously we have published information on the discovery of Coenagrion ponticum (Bartenef, 1929) in the Adygheya Republic [Shapovalov, Korotkov, 2018], but additional material allowed us to identify the species precisely as Coenagrion puella (Linnaeus, 1758).

\section{Coenagrion pulchellum (Vander Linden, 1825)}

Bartenev, 1910: Kelermesskaya; Bartenev, 1930a: Maykop (as Agrion pulchellum Lind.). $10^{x}$

MATERIAL. Maykop: Zelenstroy City District, 3.V.2017 -

6. Coenagrion scitulum (Rambur, 1842) $\beta^{\top}, 1$ ㅇ․

MATERIAL. Maykop, Voskhod City District, 15.VI.2017 -

7. Ischnura elegans (Vander Linden, 1820)

Bartenev, 1910: Kelermesskaya; Bartenev, 1930a: Maykop; Shapovalov, Korotkov, 2018: Krasny most, Botanical Garden at Adyghe State University.

MATERIAL. Maykop: Zelenstroy City District, 15.V.2015 -

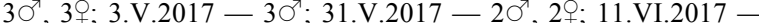

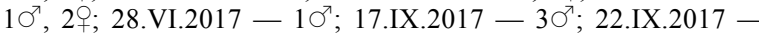
$10^{7}$; 9.IX.2017 - 10'; 22.IX.2018 - 1 \% ; environs of the infec-

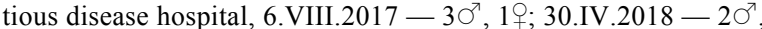
1+; Voskhod City District, 15.VI.2017 - 10'; 13.VIII.2017 1; 1.V.2018 - 10'. Maykopsky District: environs of the Krasny most, Botanical Garden at Adyghe State University, 24.VI.2016 1 ; ; 8.VI.2018 - 10'. Koshekhablsky District: environs of the Khodz, 05.VII.2017 - 107; Krasnogvardeysky District: Adamy, 8.V.2017 - 20', 1 ㅇ. Giaginsky District: Trud, Ayryum River, 18.VII.2011 - 4 $\sigma^{7}$, 1\%; Giaginskaya, Giaga River 1.V.2018 - 10 $\mathrm{O}^{\text {7 }}$; environs of the Kelermesskaya, 6.VI.2017 19 . Shovgenovsky District: environs of the Vesyoly, Ulka River, 14.V.2018-2요.

\section{Ischnura pumilio (Charpentier, 1825)}

Bartenev, 1930a: Maykop, Kurdzhips River.

\section{Erythromma najas najas (Hansemann, 1823)}

MATERIAL. Maykopsky District: environs of the Dakhovskaya, Dakh River, 16.VI.2012 - 10

At the adjacent territory of Krasnodar Krai, Krasnogvardeysky District: $2 \mathrm{~km}$ to the Belyaevsky, 8.V.2017 - 10', 1 ㅇ.

REMARKS. The species is recorded in the environs of Krasnodar [Kolosov, 1915; Bartenev, 1930a].

\section{Erythromma viridulum viridulum Charpentier, 1840}

Bartenev, 1930a: Maykop.

MATERIAL. Maykop: Zelenstroy City District, 14.VI.2016 -

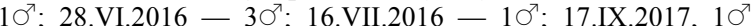
2.VII.2017 - 20'; 9.IX.2018 - $10^{7}$. environs of the Maykop, Rodnik settlement, 30.VII.2017 - $2 \sigma^{7}$. Giaginsky District: Trud Ayryum River, 18.VII.2011 - $20^{7}$.

\section{Pyrrhosoma nymphula (Sulzer, 1776)} $20^{\top}, 1$ 우.

12. Enallagma cyathigerum (Charpentier, 1840)

Bartenev, 1930a: Maykop (as Enallagma cyathigerum cyathigerum Charp.).

MATERIAL. Maykop: Zelenstroy City District, 13.V.2017 — 1 우 2.VII.2018 - 10
Family Platycnemididae

\section{Platycnemis pennipes (Pallas, 1771)}

Bartenev, 1930a: Maykop; Shapovalov, Korotkov, 2018: Krasny most, Botanical Garden at Adyghe State University.

MATERIAL. Maykop: 15.VI.2017 - 10'. Maykopsky District: environs of the Shuntuk, Shuntuk River, 25.VI.2012 - 19; environs of the Krasny most, Botanical Garden at Adyghe State University, 24.VI.2016 - 19; 8.VI.2018 - 10'; environs of the Kalinin, 27.VI.2017 - 5 $\odot^{7}, 1$. . Giaginsky District: Trud, Ayryum River, 18.VII.2011 - 80, 6웅 environs of the Kelermesskaya, Giaga River, 23.VI.2015 - 207, 8우 6.VI.2015 - 7우 7.VII.2017 3 ; ; 5.VI.2018 - 10, 1 ㅇ. Giaginskaya, 15.VI.2016 - 1\%; 6.VI.2017 - 10 , 3우 1.V.2018 - 3ㅇ. Krasnogvardeysky District: Adamy, 8.v.2017, 2ㅇ. Koshekhablsky District: environs of the Khodz, 17.VI.2016 - 1; 5.VII.2017 - 10'; Shovgenovsky District: environs of the Vesyoly, Ulka River, 14.V.2018 - 30", 2 우.

At the adjacent territory of Krasnodar Krai, Mostovsky District, environs of the Makhoshevskaya, Fars River, 16.VI.2016 4 우.

\section{Family Lestidae}

14. Chalcolestes parvidens Artobolevsky,1929

Bartenev, 1930a: Maykop (as Lestes viridis Lind.); Shapovalov, Korotkov, 2018: Krasny most, Botanical Garden at Adyghe State University.

MATERIAL. Maykop: Zelenstroy City District, 16.IX.2017 -

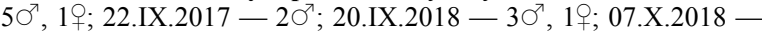
$1 \sigma^{7} ; 28 . X .2018$ - $3 \sigma^{7}$. Maykop, Voskhod City District, 26.VIII.2017 $4 \sigma^{7}$. Maykopsky District: environs of the Krasny most, Botanical Garden at Adyghe State University, 30.IX.2017 - 30, 1 웅

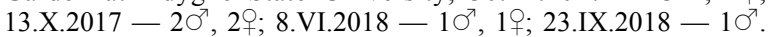
Giaginsky District: Trud, Ayryum River, 18.VII.2011 - 1오.

\section{Lestes barbarus (Fabricius, 1798)}

Bartenev, 1930a: Maykop, Kurdzhips River (as Lestes barbara Fabr.); Shapovalov, Korotkov, 2018: Krasny most, Botanical Garden at Adyghe State University.

MATERIAL. Maykopsky District: environs of the Krasny most, Botanical Garden at Adyghe State University, 30.IX.2017 $20^{\top}, 1$ ㅇ 13. X.2017 - $10^{7}$.

\section{Lestes dryas Kirby, 1890}

Bartenev, 1930a: Maykop, across the Belaya River, Kurdzhips River; Shapovalov, Korotkov, 2018: Krasny most, Botanical Garden at Adyghe State University.

MATERIAL. Maykopsky District: Khanskaya, pond, 6.VI.2012 - 2우 23.VI.2012 - 20, 1 우; environs of the Krasny most, Botanical Garden at Adyghe State University, 2.VII.2018 - 10'.

\section{Lestes sponsa (Hansemann, 1823)}

Bartenev, 1930a: Maykop, Kommunalny Khutor, the upper reaches of the Guzeripl River.

18. Lestes virens vestalis Rambur, 1842

Bartenev, 1930a: Maykop, Kurdzhips River (as Lestes virens Charp.).

\section{Sympecma fusca (Vander Linden,1820)}

Bartenev, 1930a: Maykop; Shapovalov, Korotkov, 2018: Krasny most, Botanical Garden at Adyghe State University.

MATERIAL. Maykop: Zelenstroy City District, 15.V.2015 -

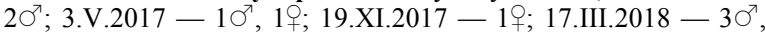

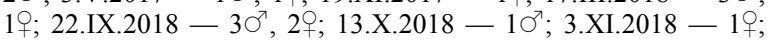
Maykop, «Mezdakh», 16.VIII.2017 - 10', 2ᄋ. Maykopsky District: environs of the Krasny most, Botanical Garden at Adyghe State University, 30.IX.2017 - 19; 13.X.2017 - 10 ${ }^{\text {7 }}$; 23.IX.2018 $-2 \sigma^{7}, 1$ ㅇ. Giaginsky District: Giaginskaya, Giaga River. 13.IV.2018 - 3O $\sigma^{\gamma}, 1+$; 1.V.2018 - $1 \sigma^{\prime}$. Krasnogvardeysky District: Adamy, 8.V.2017 - $20^{7}, 2$ ㅇ․ 


\section{ANISOPTERA}

Family Aeshnidae

20. Aeshna affinis Vander Linden, 1820

Shapovalov, Korotkov, 2018: Krasny most, Botanical Garden at Adyghe State University.

MATERIAL. Maykopsky District: environs of the Krasny most, Botanical Garden at Adyghe State University, 28.VI.2018 $4 \bigcirc^{7}$. Teuchezhsky District: Gabukay, 22.VI.2016 - 1 웅.

REMARKS. The species is recorded by A.N. Bartenev [1930b] in the adjacent territory: Krasnodar Krai, Belaya River, environs of the Tsarsky Dar villlage (now Velikovechnoye village, Belorechensky District, Krasnodar Krai).

\section{Aeshna cyanea (Müller, 1764)}

Bartenev, 1930a: Maykop; Shapovalov, Korotkov, 2018: Krasny most, Botanical Garden at Adyghe State University.

MATERIAL. Maykopsky District: environs of the Krasny most, Botanical Garden at Adyghe State University, 30.IX.2017 $10^{7}$; 13.X.2017 - 20 ; Khamyshki, 5.IX.2011 - 10 , 1 우.

\section{Aeshna juncea (Linnaeus, 1758)}

Bartenev, 1930a: Maykop.

\section{Aeshna mixta (Linnaeus, 1758)}

Bartenev, 1930a: Maykop; Meyzel, 1940: Caucasus Nature Reserve, Kisha guarding point, Guzeripl (as Aeshna coluberculus)

MATERIAL. Maykop, Zelenstroy City District, 07.X.2018 - 90" 13.X.2018 - 20 ; 3.XI.2018 - 10'. Maykopsky District: $2 \mathrm{~km}$ to the
Tulsky, canyon of the Maykopskaya River, 2.VIII.2017 - 10 $20^{\top}$.

\section{Aeshna isoceles (Müller, 1767)}

Bartenev, 1930a: Maykop.

MATERIAL. Maykop: Zelenstroy City District, 13.V.2017 $10^{\top} ; 28$. VI. $2017-10^{7}$.

\section{Anax imperator Leach, 1815}

Bartenev, 1930a: Maykop; Shapovalov, Korotkov, 2018: Krasny most, Botanical Garden at Adyghe State University.

MATERIAL. Maykop: Zelenstroy City District, 14.VI.2017 1우 28.VI.2017 - 1우 2.VI.2018 - 20', 1; 2.VII.2018 - 1웅 9.IX.2018 - $20^{7}$. Maykop, Voskhod City District, 13.VIII.2017$10^{7}$. Maykopsky District: environs of the Krasny most, Botanical Garden at Adyghe State University, 13.VI.2017 - 39 .

\section{Anax parthenope (Selys, 1839)}

Meyzel, 1940: Maykop; Caucasus Nature Reserve, Kisha guarding point.

MATERIAL. Maykop: Zelenstroy City District, 13.V.2017 1우 28.VI.2017 - 1우 16.VII.2017 - 30', 1오. Maykopsky District: Krasnooktyabrsky, 28.VI.2018, $10^{7}$. Giaginsky District: environs of the Kelermesskaya, Giaga River, 18.VI.2017 - 19.

Teuchezhsky District: Gabukay, 22.VI.2016 - 10'.

\section{Brachytron pratense (Müller, 1764)}

Shapovalov, Korotkov, 2018: Krasny most, Botanical Garden at Adyghe State University.

MATERIAL. Maykopsky District: environs of the Krasny most, Botanical Garden at Altai State University, 8.VI.2018 - $10^{5}$.

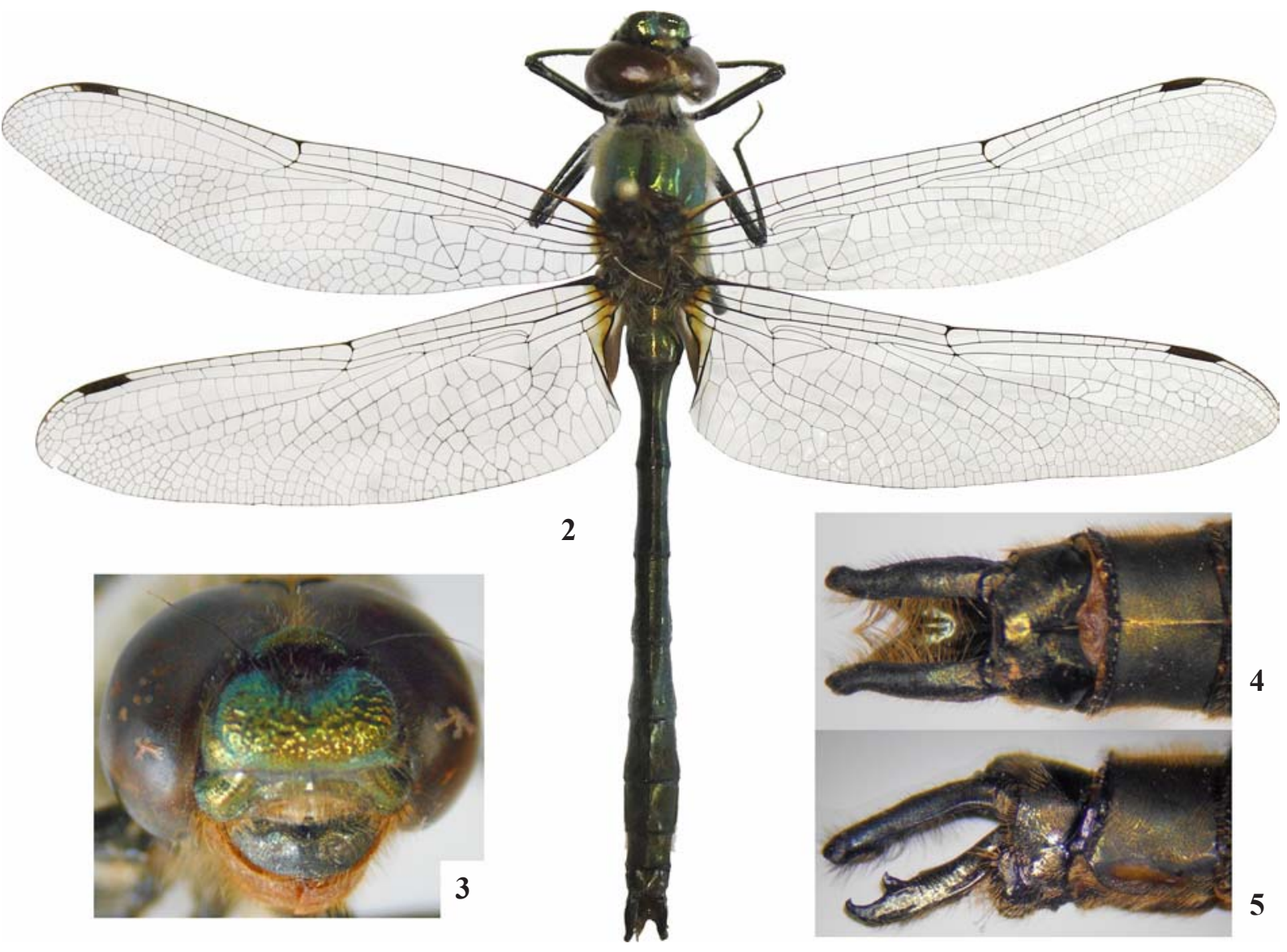

Fig. 2-5. Cordulia aenea: 2 - adult, $\bigcirc^{\Upsilon}$, dorsal view; 3 - head, frontal view; 4 - anal appendages, dorsal view; 5 - anal appendages, lateroventral view.

Рис. 2-5. Cordulia aenea: 2 - имаго, о; 3 - голова, спереди; 4 - анальные придатки, сверху; 5 - анальные придатки, сбоку. 


\section{Family Corduliidae}

28. Cordulia aenea (Linnaeus, 1758)

Figs 2-5.

MATERIAL. Maykop, Zelenstroy City District, 13.V.2017$4 \mathrm{O}^{7}$; Maykop, Voskhod City District, 14.IV.2018 - 3+; 1.V.2018 $-20^{2}$.

\section{Family Gomphidae}

29. Onychogomphus forcipatus forcipatus (Linnaeus, 1758)

Shapovalov, Korotkov, 2018: Krasny most, Botanical Garden at Adyghe State University.

MATERIAL. Maykopsky District: environs of the Dakhovskaya, 25.VI.2012 - $20^{7}$; environs of the Sevastopolskaya, 22.VI.2012 - 19. Krasnogvardeysky District: environs of the Beloye, 20.VI.2016 - 107. Koshekhablsky District: environs of the Volnoye, Laba River, 21.VI.2018 - 10 ; ; environs of the Khodz,

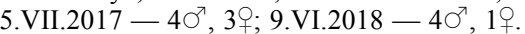

At the adjacent territory of Krasnodar Krai, Mostovsky District, environs of the Makhoshevskaya, Fars River, 16.VI.2016 $20^{2}$.

30. Onychogomphus flexuosus (Schneider, 1845) Fig. 6-9.

MATERIAL. Giaginsky District: environs of the Keler- messkaya, Giaga River, 9.VII.2018 - 40 , 2 . . Koshekhablsky District: environs of the Khodz, 5.VII.2017 - $20^{7}$

REMARKS. A.N. Bartenev [1930a] recorded the species in a basin of the Kuban River (Krasnodar, 10 July 1906). Biology of $O$. flexuosus is similar to biology of $O$. forcipatus forcipatus (Linnaeus, 1758), adults inhabit rapid rivers with pebbles on the bottom and riverside, and they can be found on pebbles near water. The presence of both species O.f. forcipatus (Linnaeus, 1758) and O.flexuosus (Schneider, 1845) was recorded in the environs of the Khodz aul (5 July 2017).

\section{Gomphus schneideri Selys, 1840}

Figs 10-15.

Shapovalov, Korotkov, 2018: Krasny most, Botanical Garden at Adyghe State University.

MATERIAL. Maykop, Zelenstroy City District, Belaya River, 28.VI.2017 - 19.

REMARKS. A.N. Bartenev [1930a] recorded a male of Gomphus vulgatissimus trans. Ad Schneideri Selys. in Maykop. In the North Caucasus, Gomphus schneideri Selys, 1840 was recorded in Dagestan [Onishko, Dunayev, 2017; Ilyina, Aliev, 2017]. In Transcaucasia, G. schneideri was recorded in Georgia [Bartenev, 1912; Reinhardt, 1992], Armenia [Tailly et al., 2004], and Azerbaijan [Skvortsov, Snegovaya, 2014].

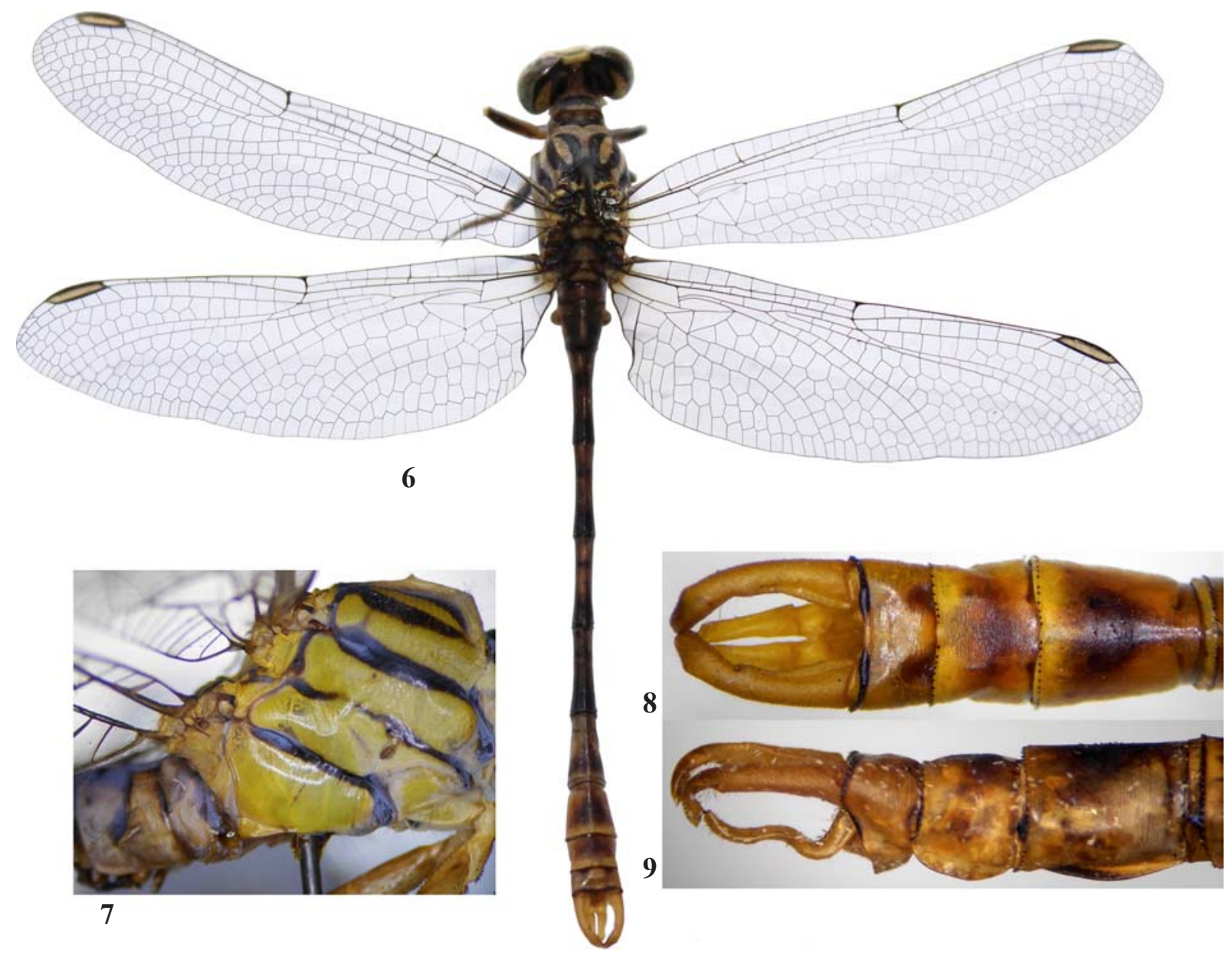

Fig. 6-9. Onychogomphus flexuosus: 6 - adult, Oै $^{7}$, dorsal view; 7 - thorax, lateral view; 8 - anal appendages, dorsal view; 9 - anal appendages, lateroventral view.

Рис. 6-9. Onychogomphus flexиosus: 6 - имаго, о7; 7 - грудь, сбоку; 8 - анальные придатки, свеху; 9 - анальные придатки, сбоку 


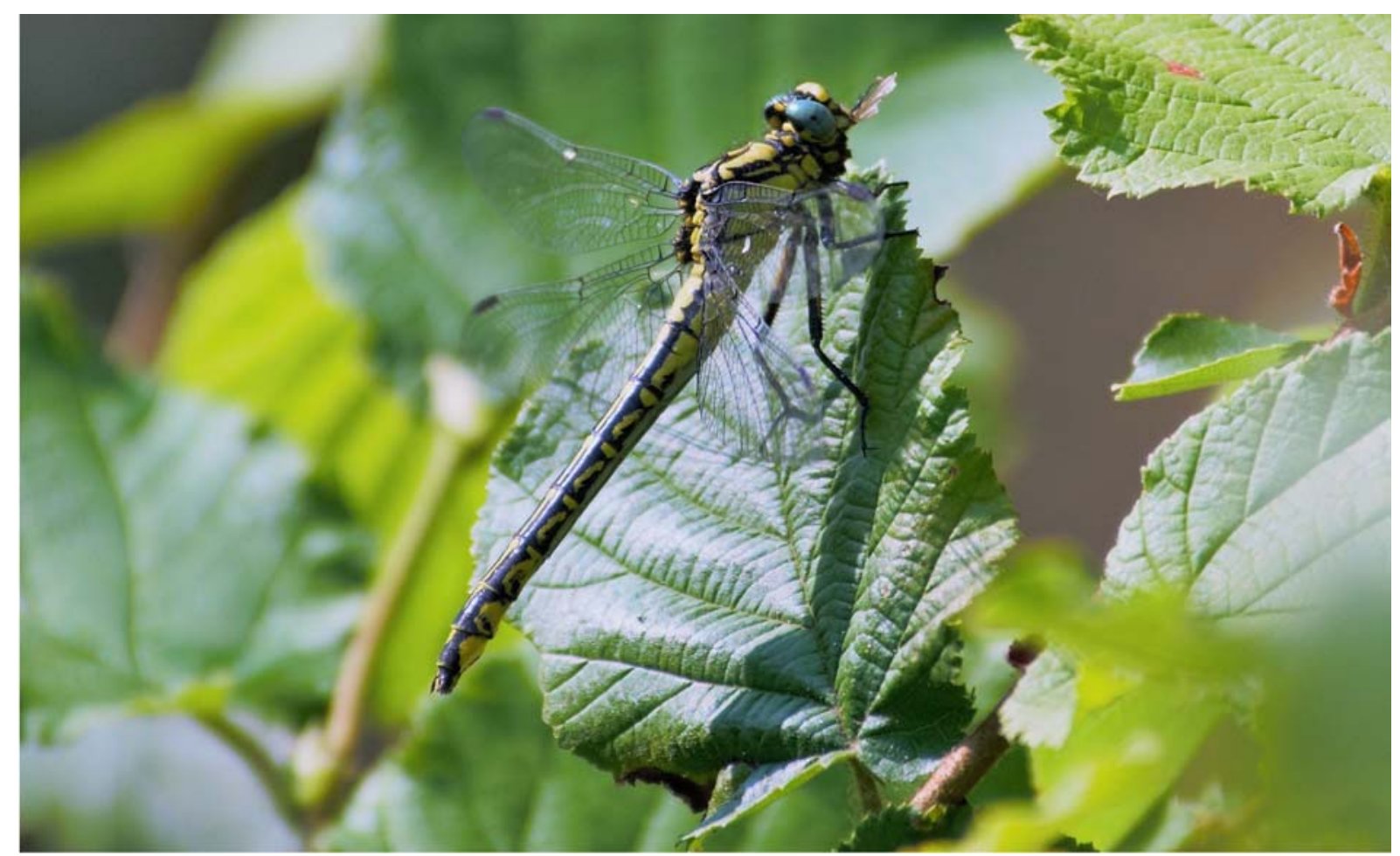

10

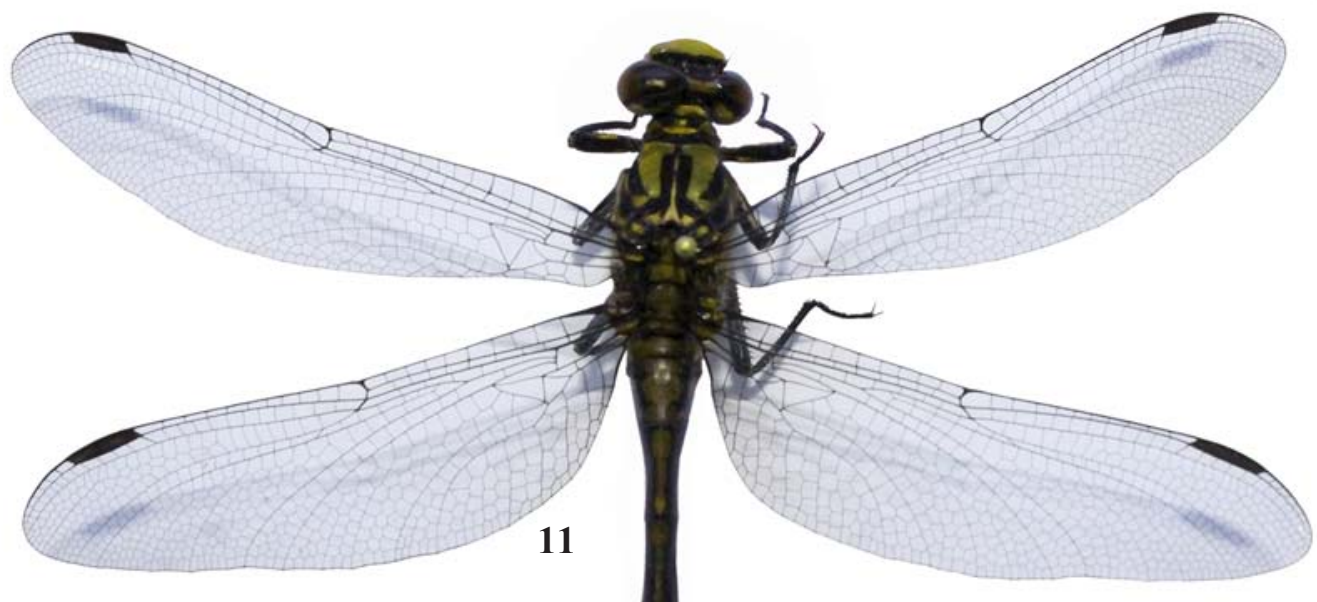

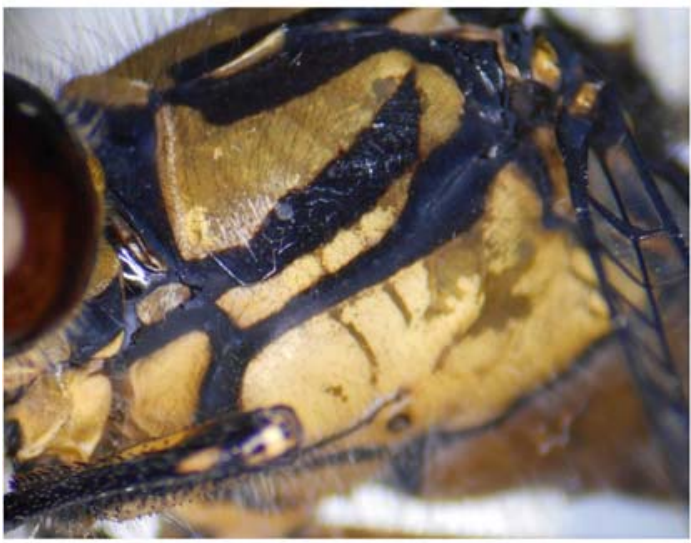

12

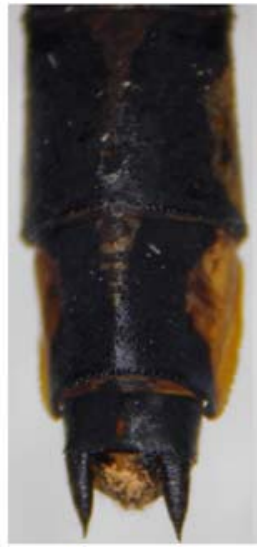

13

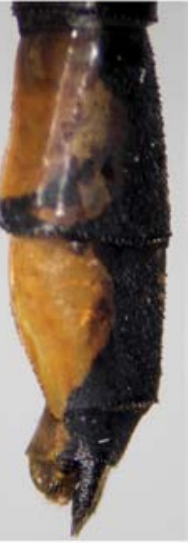

14

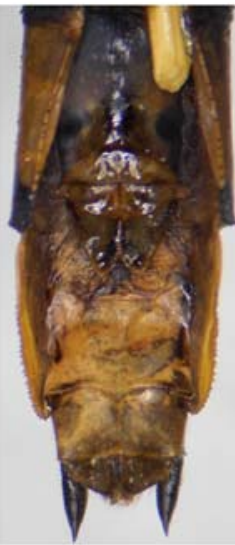

15

Fig. 10-15. Gomphus schneideri: 10 - adult, $\sigma^{\top}$, in its habitat; 11 - adult, $+; 12$ - thorax, + , lateral view; $13-15$ - abdominal apex, + . Рис. 10-15. Gomphus schneideri: 10 - имаго, О’, в природе; 11 - имаго,,+12 - грудь, ㅇ, сбоку; 13-15- конец брюшка, + . 


\section{Family Libellulidae}

32. Libellula depressa (Linnaeus, 1758)

Bartenev, 1930a: Maykop; Shapovalov, Korotkov, 2018: Krasny most, Botanical Garden at Adyghe State University.

MATERIAL. Maykop: Voskhod City District, 1.V.2018 $10^{7}, 1$; ; 7.VI.2018 - $10^{\prime}$. Maykopsky District: environs of the Krasny most, Botanical Garden at Adyghe State University, 13.VI.2017 - 5 $\sigma^{7}, 29$; 14.VI.2018 - 10'; $3 \mathrm{~km}$ to the Krasny Dagestan settlement, Kurdzhips River, 21.VI.2012 - 107. Koshekhablsky District: environs of the Khodz, 5.VII.2017 - $10^{7}$; 9.VI.2018 - 10', 1 ㅇ․

\section{Libellula fulva Müller, 1764}

Bartenev, 1930a: Maykop.

MATERIAL. Koshekhablsky District: environs of the Khodz, Khodz River, 9.VI.2018 - 30', 1 ㅇ.

\section{Orthetrum albistylum (Selys, 1848)}

Shapovalov, Korotkov, 2018: Krasny most, Botanical Garden at Adyghe State University.

MATERIAL. Maykop: Zelenstroy City District, 23.VIII.2015 -

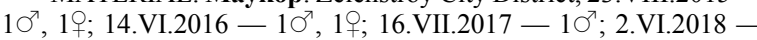

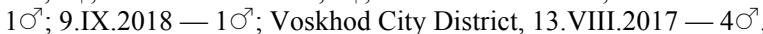
3우 7.VI.2018 - 10", 1; «Mezdakh», 16.VIII.2017 - 10". Maykopsky District: Kurdzhipskaya, 14.vi.2016 - 10, 1웅 Khanskaya, 23.VI.2012 - 10', 1웅 environs of the Krasny most, Botanical Garden at Adyghe State University, 13.VI.2017 - $4 \sigma^{7}$; 28.VI.2018 - $10^{7}$. Giaginsky District: Trud, Ayryum River,

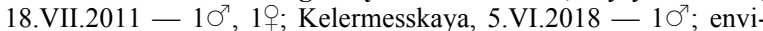
rons of the Giaginskaya, 23.VI.2015 - 29; 15.VI.2016 - $6 \sigma^{7}, 3$;

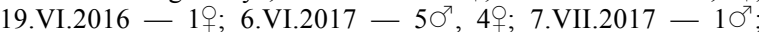
5.VI.2018 - 20', 2 ㅇ. Krasnogvardeysky District: Adamy, 28.v.2017 - 19; environs of the Elenovskoye, 20.VI.2016-207, $2+$. Koshekhablsky District: environs of the Khodz, 17.VI.2016 $1 \sigma^{\top} ; 23$. VI.2016 - $10^{\top} ;$ 5.VII.2017 - $10^{7}$.

\section{Orthetrum cancellatum (Linnaeus, 1758)} 19

MATERIAL. Maykop: Mikhaylovo City District, 15.VI.2017 -

36. Orthetrum coerulescens anceps (Fabricius, 1798)

MATERIAL. Maykop: Voskhod City District, 5.VII.2017 -

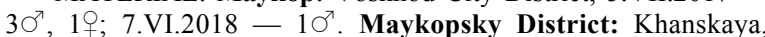
29.VI.2012 - 10'. Giaginsky District: Trud, Ayryum River, 18.VII.2011 - 10', 1; Kelermesskaya, 7.VII.2017 - $20^{7}$. Ko-

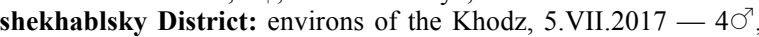
2 ㅇ.

\section{Orthetrum brunneum (Fonscolombe, 1837)}

Bartenev, 1930a: Maykop.

MATERIAL. Maykopsky District: Podgorny, 30.VI.2007 $10^{7}$; 20.VI.2016 - 10 . environs of the Dakhovskaya, 27.VI.2007 - $10^{7}$. Giaginsky District: Kelermesskaya, 23.VI.2015 - 30 , 1우 5.VI.2018 - $10^{7}$; environs of the Giaginskaya, Giaga River, 14-15.VI.2016 - 10', 1; 19.VI.2016 - 1우 5.VI.2018 - 10" Koshekhablsky District: environs of the Khodz, the Khodz River,

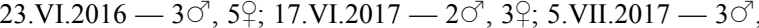
$3+$.

\section{Sympetrum fonscolombii (Selys, 1840)}

MATERIAL. Maykop: Zelenstroy City District, 16.IX.2017 -

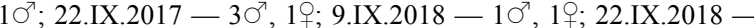
$1 \sigma^{7}, 1$; $13 . X .2018-10^{7}$.

\section{Sympetrum meridionale (Selys, 1841)}

Bartenev, 1930a: Maykop; Shapovalov, Korotkov, 2018: Krasny most, Botanical Garden at Adyghe State University.

MATERIAL. Maykop: environs of the infectious disease hospital, 6.VIII.2017 - 10'. Maykopsky District: environs of the Kalinin 29.VIII.2018 - 10', 1; Podgorny settlement, 30.VI.2007 - $10^{7}$; 20.VIII.2016 - 1 ; $; 2 \mathrm{~km}$ to the Tulsky, canyon of the Maykopskaya
River, 2.VIII.2017 - 50', 4우 ; environs of the Krasny most, Botanical Garden at Adyghe State University, 30.IX.2017 - 40 $\sigma^{7}, 3$;

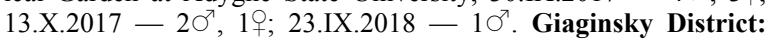
Kelermesskaya, 7.VII.2017 - 1 ㅇ.

\section{Sympetrum sanguineum (Müller, 1764)}

Bartenev, 1930a: Maykop, across the Belaya River; Shapovalov, Korotkov, 2018: Krasny most, Botanical Garden at Adyghe State University.

MATERIAL. Maykop: Mikhaylovo City District, 15.VI.2017 $30^{7}, 1$ 우 Z Zelenstroy City District, 23.VIII.2015 - 10'; 11.VI.2017-

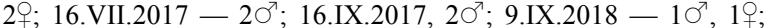
22.IX.2018 - 10; 3.XI.2018 - 20; ; Voskhod City District, 13.VIII.2017, $10^{7}$; «Mezdakh», 16.VIII.2017 - 50", 2우 ; environs of the infectious disease hospital, 6.VIII.2017 - 1우. Maykopsky District: $2 \mathrm{~km}$ to the Tulsky, canyon of the Maykopskaya River, 2.VIII.2017 - 1 . . environs of the Krasny most, Botanical Garden at Adyghe State University, 30.IX.2017 - 10', 19; 28.VI.2018 $10^{7}$. environs of the Kalinin, 27.VI.2017 - 19. Giaginsky District: Trud, Ayryum River, 18.VII.2011 - 2ㅇ. Koshekhablsky District: environs of the Khodz, 5.VII.2017 - $10^{\top}, 1$ ㅇ․

\section{Sympetrum striolatum (Charpentier, 1840)}

Shapovalov, Korotkov, 2018: Krasny most, Botanical Garden at Adyghe State University.

MATERIAL. Maykop: Zelenstroy City District, 10.VI.2016 -

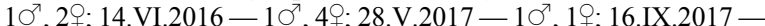

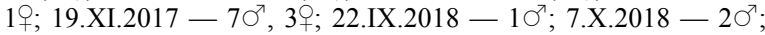

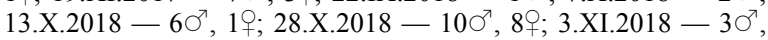

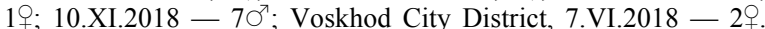
Maykopsky District: environs of the Krasny most, Botanical Garden at Adyghe State University, 8.VI.2018 - 10', 1ㅇ. Koshekhablsky District: environs of the Khodz, 5.VII.2017 - 1 웅.

42. Sympetrum vulgatum (Linnaeus, 1758)

Bartenev, 1930a: Maykop.

MATERIAL. Maykop: Zelenstroy City District, 13.X.2018 $70^{7}$. Maykopsky District: $2 \mathrm{~km}$ to the Tulsky, canyon of the Maykopskaya River, 2.VIII.2017-10'.

\section{Crocothemis erythraea (Brulle, 1832)}

MATERIAL. Maykop: Zelenstroy City District, 15.V.2015 -

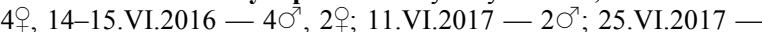

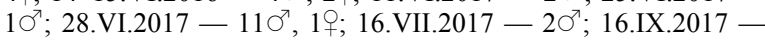

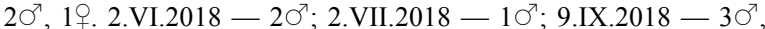

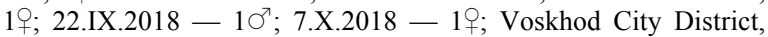

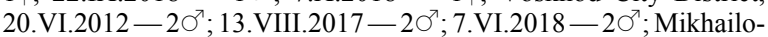
va City District, 15.VI.2017 - 20', 1ㅇ. Maykopsky District: Khanskaya, 29.V.2012 - 10', 2+; 23.VI.2012 - 1+; environs of the Dakhovskaya, Dakh River, 25.VI.2012 - $10^{7}$. Giaginsky District: Kelermesskaya, 5.VI.2018 - 2 ㅇ․

Acknowledgements. Authors are grateful to O.E. Kosterin (Novosibirsk State University, Novosibirsk) for his consultations during the preparation of the manuscript; V.V. Onishko (Moscow Region Pedagogical University, Moscow) for his consultations; M.A. Saprykin (Adyghe State University, Maykop) for his help with arrangement of odonate photographs.

\section{References}

[Atlas of the Adygheya Republic. Scientific Geographic Atlas]. T.P. Varshanina (Ed.). 2001. Maykop: GURIP “Adygheya”. 80 pp. [In Russian]

Bartenev A.N. 1910. [To the odonate fauna of the Kuban Oblast] // Russkoye Entomologicheskoye Obozreniye. Vol.12. P.27-38 [in Russian].

Bartenev A.N. 1912. [On a collection of dragonflies from the Transcaucasus of the summer 1911] // Raboty laboratorii Zoo- 
logicheskago kabineta Imperatorskago Varshavskago Universiteta. 1912 goda. P. 132-161 [in Russian, English subheadings].

Bartenev A.N. 1929. Neue Arten und Varietäten der Odonata des West-Kaukasus // Zoologischer Anzeiger. Bd.85. Nr.34. S.5468.

Bartenev A.N. 1930a. [Materials to the knowledge of Odonata of the Western Caucasus] // Trudy Severo-Kavkazskoy assotsiatsii nauchno-issledovatelskikh institutov. Vol.72. No.14. P.11-38 [in Russian, German summary, without title].

Bartenev A.N. 1930b. [On the Palearctic species of the genus Cordulegaster Leach. (Odonata)] // Raboty Severno-Kavkazskoy Gidrobiologicheskoy stantsii pri Gorskom selsko-khozyaystvennom institute. Vol.3. No.13. P.1-31 [in Russian].

Brauner A.A. 1903. [Notes on odonates] // Russkoye Entomologicheskoye Obozreniye. Vol.3. No.2. P.89-91 [in Russian]

Golub V.B., Tsurikov M.N., Prokin A.A. 2012. [Insect Collections: Collecting, Processing and Storage of Materials]. KMK Scientific Press: Moscow. 339 pp. [In Russian]

Ilina E.V., Aliev M.A. 2017. [New interesting findings of odonates in the federal protected areas of Russia of the Republic of Dagestan] // Trudy gosudarstvennogo prirodnogo zapovednika "Dagestansky". Vol.13. P.54-57 [in Russian]

Kalkman V.J. 2006. Key to the dragonflies of Turkey, including species known from Greece, Bulgaria, Lebanon, Syria, the Trans-Caucasus and Iran // Brachytron. Vol.10. No.1. P. 3-82.

Ketenchiev Kh.A., Kharitonov A.Yu. 1998. [Identification key to Caucasian Odonates]. Nalchik.120 pp. [In Russian]

Ketenchiev Kh.A., Kharitonov A.Yu., Kozminov S.G. 2016. [Caucasus as one of Mediterranean centers of the odonate species diversity (Odonata)] // Dagestan State Pedagogical University Journal. Natural and Exact Sciences. Vol.10. No.2. P.46-51 [in Russian].

Kolosov Yu.M. 1915. [Addition to the fauna of Odonata of the Kuban Oblast] // Russkoye Entomologicheskoye Obozreniye. Vol.15. No.3. P.468 [in Russian].

Kosterin O.E. 2017a. Update to Odonata of the Black Sea coast of the western Caucasus, Russia // International Dragonfly Fund Report. Vol.110. P.1-23.

Kosterin O.E. 2017b. Calopteryx virgo feminalis subsp. nov., a long known under the same name but hitherto formally nameless subspecies from the Caucasian Black Sea Coast // International Dragonfly Fund Report. Vol.107. P.45-57.

Kosterin O.E. 2018. Spring aspect of Odonata of the Abrau Peninsula, the Black Sea coast of the West Caucasus, as observed in
May 2018 // International Dragonfly Fund Report. Vol.120. P.1-13.

Kosterin O.E., Solovyev V.I. 2017. Odonata found in mid-summer 2015 and 2016 at the north-westernmost Black Sea Coast of the Caucasus, with the first record of CordulegasterpictaSelys, 1854 in Russian Federation // International Dragonfly Fund Report. Vol.107. P.1-43.

Meyzel E.D. 1940. [Preliminary annotated checklist of insects of the Caucasus Nature Reserve]. Archive of the Caucasus Nature Reserve. Archive No.132 (manuscript) [in Russian].

Onishko V.V., Dunayev E.A. 2017. [Materials to the fauna of Odonata of the "Sarykum Barkhan» of the Dagestan Nature Reserve and some other protected areas of Dagestan] // Trudy gosudarstvennogo prirodnogo zapovednika «Dagestansky». Vol.13. P.46-53 [in Russian].

Reinhardt K. 1992. Beobachtungen von Libellen an Fließgewässern in Georgien (Odonata) // Entomologische Nachrichten und Berichte. Bd.36. Hf.4. S.243-248

Shapovalov M.I., Korotkov E.A. 2018. Materials to the fauna of Odonata of the Botanical Garden at Adyghe State University // Ecosystems. Vol.16. No.46. P.94-98 [in Russian].

Skvortsov V.E. 2010. [The dragonflies of Eastern Europe and Caucasus: An illustrated guide]. Moscow: KMK Scientific Press Ltd. 623 pp. [In Russian and English]

Skvortsov V.E., Snegovaya N.Yu. 2014. Additions to the knowledge of the Odonata fauna of Azerbaijan, with six new records // Notulae odonatologicae. Vol.8. No.3. P.67-76

Stain V.Y. 2002. [Zoogeographical zoning of the North Caucasus depending on the odonate distribution (Odonata, Insecta)] // Tezisy dokladov XII s'ezda Russkogo entomologicheskogo obshchestva. St.-Peterburg. P.3-30 [in Russian].

Stain V.Y. 2003a. [Altitudinal distribution of odonates in the North Caucasus] // Fauna, questions on the ecology, morphology, and evolution of amphibiotic and aquatic insects of Russia: Materials of the II All-Russian Symposium on the amphibiotic and aquatic insects of Russia. P.218-221 [in Russian].

Stain V.Y. 2003b. [Odonates of the North Caucasus: fauna, zoogeography, and ecology]. Dissertation Abstract. Voronezh. 24 pp [In Russian]

Tailly M., Ananian V., Dumont H.J. 2004. Recent dragonfly observations in Armenia, with an updated checklist // Zoology in the Middle East. Vol.31. P.93-102.

Varshanina T.P., Mitusov D.V. 2005. [Climate resources of landscapes of the Adyghe Republic]. Maykop: Adyghe State University. 237 pp. [In Russian] 